\title{
Condiciones relacionadas con el desarrollo de septos en la cavidad abdominal de pacientes pediátricos en diálisis peritoneal
}

Patiño-García $\mathrm{G}^{1}$, Sepúlveda-Hernández $\mathrm{MT}^{2}$, Martínez-Sagredo EJ², Barrerade León $\mathrm{JC}^{3}$

\begin{abstract}
Resumen
INTRODUCCIÓN: en nuestro hospital la diálisis peritoneal es el método de sustitución renal de primera instancia. Los septos en la cavidad abdominal en niños con insuficiencia renal crónica son complicaciones por repetidos cuadros de peritonitis.
\end{abstract}

OBJETIVO: determinar las condiciones relacionadas con el desarroIlo de septos en la cavidad abdominal en pacientes pediátricos con diálisis peritoneal.

MATERIALES Y MÉTODOS: estudio observacional retrospectivo, de marzo del 2012 a octubre del 2014, incluyendo pacientes pediátricos con enfermedad renal crónica con diálisis peritoneal. Los septos fueron diagnosticados por medio de ultrasonido abdominal. Estadística inferencial con exacta de Fisher y U de Mann Whitney, asociación de riesgo con razón de momios (OR). Se incluyeron 60 pacientes en dos grupos: con septos $(n=20)$ y sin septos $(n=40)$ : sexo masculino $n=11$ $(55 \%)$ y $n=25$ (62\%); edad en años (mediana) 8 (0.5-15) y $9(0.5-16)$, respectivamente $(p=0.756)$; duración de diálisis (mediana) 12 (4-60) y $16(2-81)$ meses, también respectivamente $(p=0.653)$.

RESULTADOS: no se encontró la causa de la insuficiencia renal en 13 (65\%) de los pacientes del grupo con septos y en 25 (62\%) del grupo sin septos; factores relacionados: solución glucosada hiperosmolar $\mathrm{n}=5(25 \%)$ y $\mathrm{n}=11(28 \%)(p=0.917)$; cirugía abdominal posterior a Tenkoff $n=3(15 \%)$ y $n=7(18 \%)$; cuadros de peritonitis $n=20(100 \%)$ y $\mathrm{n}=29(72 \%)(p=0.025)$; necesidad de tratamiento sistémico $\mathrm{n}=19$ $(95 \%)$ y $n=22(55 \%)(p=0.004)$, todos los datos para los grupos con y sin septos, respectivamente.

CONCLUSIONES: dos o más eventos de peritonitis y necesidad de tratamiento sistémico son factores relacionados con el desarrollo de septos abdominales en niños con insuficiencia renal crónica en diálisis peritoneal.

PALABRAS CLAVE: peritonitis, septos abdominales, insuficiencia renal crónica.
${ }^{1}$ Servicio de Nefrología Pediátrica UMAE Hospital de Pediatría CMNO.

${ }^{2}$ Servicio de pediatría, UMAE Hospital de Pediatría CMNO, Instituto Mexicano del Seguro Social.

${ }^{3}$ División de Educación en Salud, UMAE Hospital de Pediatría CMNO. Instituto Mexicano del Seguro Social. Departamento de Reproducción Humana. Centro Universitario Ciencias de la Salud. Universidad de Guadalajara, Guadalajara, Jalisco.

Servicio de Nefrología Pediátrica, UMAE Hospital de Pediatría, CMNO, Instituto Mexicano del Seguro Social. Guadalajara, Jalisco, México.

Recibido: 3 de diciembre del 2015

Aceptado: 8 de junio del 2016

Correspondencia

Dr. Juan Carlos Barrera de León

jcbarrer@hotmail.com

Este artículo debe citarse como

Patiño-García G, Sepúlveda-Hernández MT, Martínez-Sagredo EJ, Barrera-de León JC. Condiciones relacionadas con el desarrollo de septos en la cavidad abdominal de pacientes pediátricos en diálisis peritoneal. Acta Pediatr Mex. 2017;38(1):17-25. 


\section{Conditions related with the development of septae in the abdominal cavity of pediatric patients in peritoneal dialysis.}

Patiño-García G ${ }^{1}$, Sepúlveda-Hernández $\mathrm{MT}^{2}$, Martínez-Sagredo EJ², Barrerade León JC ${ }^{3}$

\begin{abstract}
BACKGROUND: At our hospital, peritoneal dialysis is a first-instance renal substitution method. Septae in the abdominal cavity of children with renal insufficiency comprise complications for repeated manifestations of peritonitis.

OBJECTIVE: To determine conditions related with the development of septae in the abdominal cavity of pediatric patients with peritoneal dialysis.

MATERIALS AND METHODS: Observational retrospective study from March 2012 to October 2014 that included pediatric patients with chronic renal disease with peritoneal dialysis. The presence of septae was determined by abdominal UltraSound (US). Inferential statistics with Fisher exact test and Mann-Whitney $U$, risk association with Odds Ratio (OR). Sixty patients in two groups: the group with septae $n=20$, and the group without septae $n=40$. Masculine gender $n=11(55 \%)$ vs. $n=25$ (62\%): age (years), median 8 years (range, $0.5-15$ years) vs. 9 years (range, $0.5-16) p=0.756$; median dialysis duration 12 months (range, 4-60 months) vs. 16 months (range, $2-81$ months) $p=0.653$.

RESULTS: Etiology of undetermined renal insufficiency $n=13$ (65\%) vs. $n=25(62 \%)$, and related factors: hyperosmolar glycosylated solution $n=5(25 \%)$ vs. $n=11(28 \%) p=0.917$; abdominal surgery after Tenckoff $n=3(15 \%)$ vs. $n=7(18 \%)$; clinical manifestations of peritonitis $n=20$ $(100 \%)$ vs. $n=29(72 \%) p=0.025$, and systemic treatment $n=19(95 \%)$ vs. $n=22(55 \%)$ in the groups with and without septae $(p=0.004)$.
\end{abstract}

CONCLUSIONS: Number of events of peritonitis and systemic treatment are factors related with the development of abdominal septae in children with chronic renal insufficiency in peritoneal dialysis.

KEYWORDS: peritonitis; encapsulating peritoneal sclerosis; chronic kidney insufficiency

\begin{abstract}
${ }^{1}$ Servicio de Nefrología Pediátrica UMAE Hospital de Pediatría CMNO.

${ }^{2}$ Servicio de pediatría, UMAE Hospital de Pediatría CMNO, Instituto Mexicano del Seguro Social.

${ }^{3}$ División de Educación en Salud, UMAE Hospital de Pediatría CMNO. Instituto Mexicano del Seguro Social. Departamento de Reproducción Humana. Centro Universitario Ciencias de la Salud. Universidad de Guadalajara, Guadalajara, Jalisco.
\end{abstract}

Servicio de Nefrología Pediátrica, UMAE Hospital de Pediatría, CMNO, Instituto Mexicano del Seguro Social. Guadalajara, Jalisco, México.

Correspondence

Dr. Juan Carlos Barrera de León jcbarrer@hotmail.com

\section{INTRODUCCIÓN}

La enfermedad renal crónica es un deterioro lento de la función renal, la etiología es variada y de gran impacto en la salud. Se estima que en México existen 70 mil personas con esta enfermedad, de los cuales el $20 \%$ son menores de 18 años y $90 \%$ están en tratamiento sustitutivo 
de diálisis peritoneal. El Instituto Mexicano del Seguro Social trata al $80 \%$ de los pacientes en nuestro país. ${ }^{1,2}$

Existen tres terapias de reemplazo renal: la hemodiálisis, el trasplante renal y la diálisis peritoneal; esta última utiliza la cavidad peritoneal como filtro para actuar como riñón artificial y se indica cuando la filtración glomerular es igual o menor a $15 \mathrm{~mL} / \mathrm{min} / 1.73 \mathrm{~m}^{2}$ (superficie corporal real). ${ }^{3}$ Su objetivo es permitir un intercambio de solutos y agua a través de la membrana que separa dos compartimientos líquidos permitiendo la difusión, ultrafiltración y absorción de dichos solutos. Hay dos modalidades: la diálisis peritoneal continua ambulatoria y la diálisis peritoneal automática. $^{4}$

Las infecciones peritoneales tienen alta morbilidad y mortalidad, ocurren 0.5 a 0.71 episodios por paciente por año. Las alteraciones comunes son la comunicación no natural con el exterior por la introducción de un catéter y la reincorporación continua con el equipo de diálisis dentro de la cavidad abdominal. ${ }^{5}$

Una de las complicaciones de la diálisis peritoneal asociada con los cuadros repetitivos de peritonitis es la esclerosis encapsulada del peritoneo, cuya incidencia varía de 0.7 a $7.3 \%$; su causa se desconoce. ${ }^{6}$ Los septos en la cavidad abdominal se pueden presentar como complicación de cuadros repetidos de peritonitis, aunque se consideran de origen multifactorial, asociándoseles con otros factores como la duración del tratamiento de reemplazo sustitutivo, las infecciones secundarias por Stafiloccoccus aureus y coagulasa negativos, así como exposición a soluciones hipertónicas que contienen glucosa. ${ }^{3}$

Los cuadros bacterianos refractarios y recurrentes propician que se sinteticen enzimas que convierten fibrinógeno en fibrina, lo que promueve adherencias intestinales. La extravasación de líquidos ocasiona edema visible con infiltrados de líquidos dentro de los tejidos subcutáneos, ocasionando falla en el drenaje de líquidos y equilibrios positivos. ${ }^{7}$

La presentación clínica habitual es la disfunción de la diálisis peritoneal, así como síntomas gástricos, en ocasiones tan severos como los de una obstrucción intestinal. ${ }^{8}$ Los estudios de imagen son de gran ayuda para el diagnóstico y la identificación de complicaciones inherentes, así como para su localización exacta. El ultrasonido es un método no invasivo que determina su presencia y localización; sin embargo, en algunos casos se requiere una tomografía computarizada que se ha definido como el método diagnóstico más útil en pacientes con mayor estado de inflamación. ${ }^{8}$

El tratamiento consiste en suspender la diálisis peritoneal, cambiar de método de sustitución renal a hemodiálisis. En casos graves, con sospecha de obstrucción intestinal, se realiza una laparotomía exploradora con lisis de los septos y resolución del cuadro obstructivo. ${ }^{8,9}$

\section{OBJETIVO}

Determinar los factores de riesgo asociados con el desarrollo de septos en la cavidad abdominal, secundarios a peritonitis, en pacientes pediátricos con diálisis peritoneal.

\section{MATERIAL Y MÉTODOS}

\section{Diseño de estudio}

Estudio observacional, retrospectivo, realizado de marzo del 2012 a octubre del 2014, en niños del Servicio de Nefrología Pediátrica con enfermedad renal crónica, en terapia sustitutiva de diálisis peritoneal en un hospital de referencia de tercer nivel del país. 


\section{Criterios de selección}

Se incluyeron niños de 1 a 16 años, tratados en el servicio de nefrología pediátrica en los programas de diálisis peritoneal continua ambulatoria o diálisis peritoneal automática de la unidad. Se excluyeron pacientes con expedientes incompletos.

\section{Desarrollo del estudio}

Se revisaron las notas de evolución de los expedientes clínicos físicos y electrónicos, vaciando los datos en una hoja de recolección de datos. Se identificó a los pacientes con insuficiencia renal en terapia de sustitución. Se formaron dos grupos: grupo de casos con pacientes con septos en la cavidad abdominal y grupo control de pacientes sin septos en la cavidad abdominal.

\section{Septos en la cavidad abdominal}

Se definió al septo en la cavidad abdominal como una complicación de los cuadros repetidos de peritonitis, en ocasiones con adherencias intestinales y extravasación de líquidos, edema visible con infiltraciones de fluidos dentro de tejidos subcutáneos ocasionando falla en el drenaje de líquidos y equilibrios positivos. Se diagnosticó con ultrasonido abdominal (transductor convexo marca Toshiba de 7.5 MHz) de baja frecuencia y alta penetración. El paciente se colocó en decúbito dorsal, de preferencia con líquido de diálisis para mejor visualización de posibles septos.

\section{Análisis estadístico}

El análisis descriptivo de variables cualitativas se realizó con frecuencias y porcentajes; las variables cuantitativas se analizaron con medianas y rangos mínimo y máximo. Para estadística inferencial, en el caso de variables categóricas, la comparación de proporciones se realizó con prueba exacta de Fisher. La comparación de variables cuantitativas se llevó a cabo con $U$ de Mann Whitney. La asociación de riesgo se determinó con razón de momios (OR). El análisis de los datos se realizó con el paquete estadístico SPSS versión 21.0. Se consideró diferencia estadísticamente significativa un valor de $p<0.05$.

\section{Tamaño de la muestra}

No se realizó cálculo de tamaño de muestra ya que se incluyó a todos los pacientes que desarrollaron septos en la cavidad peritoneal, en el periodo de estudio, y se compararon con el doble de pacientes sin septos abdominales como grupo control.

\section{Consideraciones éticas}

Se trata de un estudio con riesgo menor al mínimo, según la Ley General de Salud, por lo que no requirió consentimiento informado. El trabajo fue aceptado por el comité local de investigación y ética del hospital con número de folio R-2014-1302-052.

\section{RESULTADOS}

Se evaluaron 60 expedientes médicos de niños, con diagnóstico de enfermedad renal crónica en tratamiento sustitutivo con diálisis peritoneal, divididos en dos grupos: el primero de 20 pacientes que desarrollaron septos y el segundo grupo con 40 que no desarrollaron septos. La distribución por sexo sexo fue similar en ambos grupos: sexo masculino 11 (55\%) en el grupo en estudio y 25 (62\%) en el grupo de casos. El intervalo de edad fue de 0.5 a 15 años en el grupo con septos; en el grupo sin septos el rango fue de 0.5 a 16 años. La mayoría de niños fueron tratados con la modalidad de diálisis peritoneal automática en ambos grupos: 70 y $80 \%$, respectivamente. El tiempo de tratamiento con diálisis peritoneal en el primer grupo tuvo una mediana de 12 meses 
en comparación con los 16 meses del segundo grupo $(p=0.653)$ Cuadro 1.

En el grupo de niños con septos en 13 (65\%) no se encontró una causa que explicara el diagnóstico, así como en 25 (62\%) del grupo sin septos. Las causas más frecuentes encontradas fueron atrofia renal y uropatías (Cuadro 2). La comorbilidad más

Cuadro 1. Características demográficas de los pacientes

\begin{tabular}{|l|c|c|c|} 
& $\begin{array}{c}\text { Grupo } \\
\text { con septos } \\
\mathbf{n = 2 0}\end{array}$ & $\begin{array}{c}\text { Grupo } \\
\text { sin septos } \\
\mathbf{n = 4 0}\end{array}$ & $\begin{array}{c}\text { Valor } \\
\boldsymbol{p}\end{array}$ \\
\hline Sexo & \multicolumn{3}{|c}{0.589} \\
\hline Masculino, n (\%) & $11(55)$ & $25(62)$ & \\
\hline Femenino, n (\%) & $9(45)$ & $15(38)$ & \\
\hline Edad en años, Med (rango) & $8(0.5-15)$ & $9(0.5-16)$ & 0.756 \\
\hline Tipo de diálisis peritoneal & & & 0.518 \\
\hline DPCA, n (\%) & $6(30)$ & $8(20)$ & \\
\hline DPA, n (\%) & $14(70)$ & $32(80)$ & \\
\hline Duración diálisis, & $12(4-60)$ & $16(2-81)$ & 0.653 \\
Med (rango) & $7(35)$ & $13(32)$ & \\
\hline $0-9$ meses, n (\%) & $7(35)$ & $12(30)$ & \\
\hline 10-19 meses, n (\%) & $1(5)$ & $5(12)$ & \\
\hline 20-29 meses, n (\%) & $5(25)$ & $10(25)$ & \\
\hline 30 o mas meses, n(\%) & &
\end{tabular}

Prueba exacta de Fisher para variables categóricas y $U$ de Mann Whitney para variables numéricas. n: número; Med: mediana; DPCA: diálisis peritoneal continua ambulatoria; DPA: diálisis peritoneal automática.

Cuadro 2. Causas de la insuficiencia renal

\begin{tabular}{|l|c|c|}
\hline Causa & $\begin{array}{c}\text { Grupo } \\
\text { con septos } \\
\mathbf{n = 2 0}\end{array}$ & $\begin{array}{c}\text { Grupo sin } \\
\text { septos } \\
\mathbf{n = 4 0}\end{array}$ \\
\hline Indeterminada, n (\%) & $13(65)$ & $25(62)$ \\
\hline Atrofia renal, n (\%) & $2(10)$ & $7(17)$ \\
\hline Agenesia renal, n (\%) & $1(5)$ & 0 \\
\hline Glomerulopatía crónica, n (\%) & $3(15)$ & $1(3)$ \\
\hline Uropatías, n (\%) & $1(5)$ & $5(12)$ \\
\hline Pielonefritis crónica, n (\%) & 0 & $1(3)$ \\
\hline Isquemia, n (\%) & 0 & $1(3)$
\end{tabular}

frecuente en ambos grupos fue la hipertensión arterial sistémica: 8 (40\%) para el grupo con septos y $15(38 \%)$ para el grupo sin septos. La asociación de hipertensión arterial y cardiopatía congénita se presentó en 1 paciente (5\%) en el grupo con septos. En el grupo sin septos dos pacientes (5\%) tuvieron estreñimiento, 2 (5\%) pancreatitis y 1 (2\%) derrame pericárdico (Cuadro 3).

El Cuadro 4 muestra la relación del uso de soluciones hiperosmolares en 5 niños $(25 \%)$ del primer grupo comparado con 11 (28\%) del segundo. El tiempo de evolución de la peritonitis no mostró diferencia entre grupos. No se identificaron pacientes, en el grupo de estudio, de cirugías abdominales previas a la colocación del catéter de Tenckoff. Cirugías abdominales posteriores a la colocación del catéter y recambios del mismo se identificaron en 3 niños (15\%) para un recambio de catéter en el grupo con septos y 7 (18\%) para el grupo sin septos. No se hallaron otras etiologías de cirugías abdominales o que se manipulara a nivel peritoneal. El germen aislado con mayor frecuencia en el grupo con septos fue Staphylococcus aureus en 9 (45\%), Pseudomonas aureginosa 3 (15\%) y Staphylococcus

Cuadro 3. Morbilidad asociada en pacientes pediátricos portadores de insuficiencia renal crónica manejados con diálisis peritoneal

\begin{tabular}{|c|c|c|}
\hline Morbilidad & $\begin{array}{c}\text { Grupo con } \\
\text { septos } \\
\mathbf{n}=\mathbf{2 0}\end{array}$ & $\begin{array}{c}\text { Grupo sin } \\
\text { septos } \\
n=40\end{array}$ \\
\hline Ninguna, $\mathrm{n}(\%)$ & $10(50)$ & $17(43)$ \\
\hline $\begin{array}{l}\text { Hipertensión arterial sistémica, } \\
\mathrm{n}(\%)\end{array}$ & $8(40)$ & $15(38)$ \\
\hline $\begin{array}{l}\text { HAS + cardiomiopatía dilatada, } \\
\mathrm{n}(\%)\end{array}$ & $1(5)$ & 0 \\
\hline $\begin{array}{l}\text { HAS + cardiopatía congénita, } \\
\mathrm{n}(\%)\end{array}$ & $1(5)$ & $3(7)$ \\
\hline Estreñimiento, n(\%) & 0 & $2(5)$ \\
\hline Pancreatitis, n(\%) & 0 & $2(5)$ \\
\hline Derrame pericárdico, n(\%) & 0 & $1(2)$ \\
\hline
\end{tabular}

n: número; HAS: hipertensión arterial sistémica. 
Cuadro 4. Características de la peritonitis en pacientes pediátricos portadores de afección renal crónica por grupo de presentación

\begin{tabular}{|c|c|c|c|}
\hline & $\begin{array}{c}\text { Grupo con septos } \\
n=20\end{array}$ & $\begin{array}{c}\text { Grupo sin septos } \\
n=40\end{array}$ & Valor $p$ \\
\hline Solución glucosada hiperosmolar, n (\%) & $5(25)$ & $11(28)$ & 0.917 \\
\hline Tiempo de presentación de septos, Med (rango) & $0.00(0-1)$ & $0.00(0-1)$ & 0.980 \\
\hline Menor a un mes, n (\%) & $15(75)$ & $30(75)$ & \\
\hline Un mes o más, n (\%) & $5(25)$ & $10(25)$ & \\
\hline Cirugía abdominal posterior a Tenckoff, n (\%) & $3(15)$ & $7(18)$ & 0.902 \\
\hline Recambios de catéter & & & 0.392 \\
\hline $1, \mathrm{n}(\%)$ & $3(15)$ & $6(15)$ & \\
\hline $2, \mathrm{n}(\%)$ & 0 & $3(7)$ & \\
\hline Cuadros de peritonitis, $\mathrm{n}(\%)$ & $20(100)$ & $29(72)$ & 0.025 \\
\hline \multicolumn{4}{|l|}{ Eventos de peritonitis } \\
\hline 1 evento, n (\%) & $2(10)$ & $15(52)$ & \\
\hline 2 eventos, n (\%) & $9(45)$ & $8(37)$ & \\
\hline 3 eventos, n (\%) & $6(30)$ & $2(7)$ & \\
\hline 4 eventos, $\mathrm{n}(\%)$ & $2(10)$ & $2(7)$ & \\
\hline 5 o más eventos, n (\%) & $1(5)$ & $2(7)$ & \\
\hline \multicolumn{4}{|l|}{ Germen aislado en cultivo peritoneal, n (\%) } \\
\hline Stafilococo aureus & $9(45)$ & $9(22)$ & \\
\hline Pseudomona aureoginosa & $3(15)$ & $5(12)$ & \\
\hline Stafilococo epidermidis & $2(10)$ & $1(2)$ & \\
\hline Necesidad de tratamiento sistémico, n (\%) & $19(95)$ & $22(55)$ & 0.004 \\
\hline Necesidad de tratamiento mas 14 días, n (\%) & $6(30)$ & $8(20)$ & 0.443 \\
\hline
\end{tabular}

Prueba exacta de Fisher para variables categóricas.

epidermidis $2(10 \%)$, no se aisló germen en 6 niños. En el grupo sin septos los gérmenes que con mayor frecuencia fueron Staphylococcus aureus en 9 (22\%) y Pseudomonas aureginosa 5 (12\%). Los pacientes que ameritaron tratamiento sistémico fueron 19 pacientes (95\%); en el grupo con septos comparado con 22 pacientes (55\%) en el grupo $\sin \operatorname{septos}(p=0.004)$.

La asociación para la presentación de septos en nuestro estudio fueron dos o más eventos de peritonitis; se presentaron 16 veces más probabilidades de presentar septos en la cavidad abdominal, con un OR de 16.71 (IC 95\%: 3.31-
82.69). Tres o mas eventos OR 4.63 (IC 95\%: 1.34-15.96). La necesidad de aplicación de tratamiento sistémico para el manejo de la peritonitis tuvo un OR de 15.54 (IC 95\%: 1.89127.60). El germen más frecuente dentro de los pacientes del grupo de casos y control es el S. aureus, pero sin significancia al considerar el intervalo de confianza. Al analizar la asociación con otras variables no se presentó significancia. Las variables analizadas fueron las soluciones hiperosmolares dentro de la diálisis peritoneal, la etiología de la enfermedad renal crónica y la cirugía abdominal posterior a la colocación de catéter de Tenckoff (Cuadro 5). 
Cuadro 5. Asociación de factores relacionados con las características de la peritonitis con presencia de septos en pacientes pediátricos

\begin{tabular}{|l|c|c|}
\hline Variable & OR & IC $95 \%$ \\
\hline $\begin{array}{l}\text { Número de eventos de peritonitis } \\
\text { 1 evento de peritonitis }\end{array}$ & 0.18 & $0.03-0.91$ \\
\hline 2 o mas eventos de peritonitis & 16.71 & $3.31-82.69$ \\
\hline o mas eventos de peritonitis & 4.63 & $1.34-15.96$ \\
\hline Germen aislado & & \\
\hline \multicolumn{1}{|l}{ Staphylococcus aureus } & 2.81 & $0.89-8.91$ \\
\hline $\begin{array}{l}\text { Pseudomona aureginosa } \\
\text { Solución hiperosmolar }\end{array}$ & 1.23 & $0.26-5.78$ \\
\hline $\begin{array}{l}\text { Modalidad DPCA } \\
\text { Cirugía abdominal posterior }\end{array}$ & 1.18 & $0.35-3.90$ \\
\hline $\begin{array}{l}\text { T Tenckoff } \\
\text { Necesidad de tratamiento sistémico }\end{array}$ & 15.54 & $1.89-127.6$ \\
\hline $\begin{array}{l}\text { Duración tratamiento mayor } \\
\text { 14 días }\end{array}$ & 2.00 & $0.57-6.97$ \\
\hline
\end{tabular}

IRC: insuficiencia renal crónica; DPCA: diálisis peritoneal continua ambulatoria; OR: razón de momios; IC 95\%: intervalo de confianza de $95 \%$.

\section{DISCUSIÓN}

En la actualidad se ha referido un creciente número de pacientes con enfermedad renal crónica y deterioro de la función renal de etiología variada. En México se reportan aproximadamente 70 mil personas con dicha enfermedad; de éstas, cerca de $90 \%$ se encuentran en tratamiento con diálisis peritoneal. ${ }^{10}$ Se estima que los septos abdominales secundarios a diálisis son un problema importante en la población, ya que condicionan que la cavidad peritoneal no funcione adecuadamente y obligue a cambiar de modalidad de reemplazo renal.

Los factores de riesgo para el desarrollo de septos en la cavidad abdominal no son claros. En la literatura se comentan múltiples factores que podrían contribuir a su desarrollo; se refiere que la etiología de la enfermedad renal crónica es distinta en adultos, lactantes y preescolares.
Para los primeros se han descrito, principalmente, las anomalías congénitas del riñón y de las vías urinarias; mientras que para los escolares y adolescentes las enfermedades glomerulares primarias o secundarias son las más comunes. En nuestro estudio no se pudo relacionar una etiología específica, refiriéndose como indeterminada en $65 \%$ para los casos y $60 \%$ para los controles, seguida de glomerulopatías, atrofia renal y uropatías.

Los septos en la cavidad abdominal pueden ser una complicación de cuadros repetidos de peritonitis, lo que representa una de las principales complicaciones infecciosas de los pacientes con insuficiencia renal crónica terminal y conlleva el riesgo de secuelas como fibrosis y adherencias peritoneales que pueden comprometer la eficacia de la diálisis de la membrana peritoneal. ${ }^{3}$ Sin embargo, también se ha visto que existe una etiología multifactorial, así como la duración del tratamiento de remplazo sustitutivo o infecciones secundarias a Staphilococcus aureus y coagulasa negativa. Sandi y sus colaboradores comentan que algunos de los mayores factores de riesgo son la duración de diálisis peritoneal, episodios recurrentes de peritonitis y la exposición a soluciones hipertónicas que contengan glucosa. ${ }^{3}$

En este estudio se analizaron varios factores asociados con el desarrollo de septos en la cavidad peritoneal. El primero de ellos fue el tipo de diálisis peritoneal, método utilizado inicialmente en su tratamiento, ya que permite al paciente llevar a cabo una vida lo más cercana a lo normal utilizando una máquina cicladora nocturna (diálisis peritoneal automática).

La infección peritoneal ocasiona fibrosis en la cavidad abdominal; las infecciones repetidas ocasionan peritonitis refractaria o resistente a tratamiento, lo que a su vez genera cuadros repetitivos de peritonitis y contribuye a la formación de septos en la cavidad peritoneal. Una vez que 
se detecta que el paciente desarrolla septos en la cavidad abdominal es indicación absoluta para el retiro de la diálisis peritoneal y para el cambio de modalidad. En nuestro estudio se encontró que todos los pacientes del grupo de niños que desarrollaron septos tuvieron peritonitis, y al presentar más de 4 eventos de este tipo se asoció significativamente a desarrollar septos. ${ }^{11}$ Los microorganismos que se asocian a la diálisis peritoneal son el Staphylococcus aureus y el Staphylococcus epidermidis; sin embargo, los bacilos gramnegativos son responsables de una tercera.

Los cuadros repetidos de peritonitis por estas bacterias sintetizan enzimas que convierten el fibrinógeno en fibrina, lo que promueve las adherencias intestinales, así como una extravasación de líquidos ocasionando edema visible con infiltraciones de fluidos dentro de los tejidos subcutáneos, ocasionando falla en el drenaje de fluidos y equilibrios positivos. ${ }^{12}$ En nuestro estudio el germen que se aisló con mayor frecuencia fue el Staphylococcus aureus en ambos grupos, sin ser un factor concluyente asociado a la presencia de septos.

El ultrasonido es el método no invasivo que se utiliza con mayor frecuencia para detectar septos. Se han descrito situaciones donde el uso de otros métodos, como la tomografía computarizada, es útil, generalmente en pacientes con mayor estado de inflamación. En nuestro estudio se utilizó en todos los casos el ultrasonido abdominal, requiriendo sólo en un paciente realizar tomografía computarizada. El ultrasonido se ha referido como un método útil, rápido, eficaz, no invasivo y de fácil acceso en el medio hospitalario. ${ }^{13}$

El factor de riesgo más significativo es el tratamiento utilizado durante los eventos de peritonitis. En la literatura se comenta que el tratamiento ideal debe ser el local o intraperitoneal de forma empírica ante la sospecha de peritonitis. La cefalosporina de primera generación y la ceftazidima son las que con mayor frecuencia se utilizan. ${ }^{14} \mathrm{~A}$ pesar de tener una diferencia amplia en este resultado, este hallazgo hace pensar que el tratamiento intraperitoneal es el adecuado para reducir los casos de peritonitis recurrente o refractaria, disminuyendo la incidencia de infecciones y así evitar complicaciones por septos abdominales.

Los factores de riesgo que se comentan en la literatura, para el desarrollo de septos, como la duración del tratamiento, las cirugías abdominales posteriores a la colocación de catéter de Tenckoff y soluciones hiperosmolares, en nuestro estudio no representaron significancia estadística para el desarrollo de los septos abdominales; sin embargo, es importante reconocer que el número de pacientes limita las conclusiones al respecto. No obstante, corroboramos que efectivamente los eventos de peritonitis son causa importante del desarrollo de los septos abdominales. Así mismo, se encontró que a mayor número de eventos de peritonitis más riesgo de desarrollar septos; en este caso más de 2 cuadros por paciente contribuyen a su formación.

El germen identificado para el grupo de casos y controles fue el Staphylococcus aureus, seguido de la Pseudomona aureginosa, para ambos grupos. El tratamiento sistémico comparado con el intraperitoneal contribuye como factor de riesgo para el desarrollo de septos abdominales. No se encontraron como factores de riesgo el uso de soluciones hipertónicas, las cirugías posteriores a la colocación del catéter de Tenckoff o la duración del tratamiento de sustitución.

\section{REFERENCIAS}

1. López-Cervantes $\mathrm{M}$, Rojas Rusell ME, Tirado-Gómez $\mathrm{CL}$, Durán-Arenas L, Pacheco-Domínguez RL, Venado-Estrada $A A$, et al. Enfermedad renal crónica y su atención mediante tratamiento sustitutivo en México. México DF: Facultad de Medicina, Universidad Autónoma de México. 2010:19-38. 
Patiño-García G et al. Riesgo de septos en la cavidad abdominal

2. Méndez-Durán A, Méndez-Bueno JF, Tapia-Yañez T, Miñoz-Montes A, Aguilar-Sánchez L. Epidemiología de la insuficiencia renal crónica en México. Dial Traspl. 2010;31:7-11.

3. Sandi MS, Romay NM, Ríos CN, Kuszmiruk AC. Diálisis peritoneal: Complicaciones más frecuentes. Revista de posgrado de la VI cátedra de Medicina. 2010;199:16-21.

4. Rabindranath KS, Adams J, Ali TZ, Daly C, Vale L, Macleod AM. Automated vs continuous ambulatory peritoneal dialysis: a systematic review of randomized controlled trials. Nephrol Dial Transplant. 2007;22:2991-8.

5. Morales Aguirre JJ, Aguelles Guerrero AG. Peritonitis secundaria a diálisis en pacientes con insuficiencia renal crónica: reporte de seis años en un hospital de tercer nivel. Bol Med Hosp Infant Mex. 2007;64:221-230.

6. Fernández-Villa-Fernández A, Reguera-Álvarez R, GonzálezDíaz I. Esclerosis peritoneal: análisis de dos casos. Rev Soc Esp Enferm Nefrol. 2009;12:164-166.

7. Hendriks MP, de Sevaux RG, Hilbrands LB. Encapsulating peritoneal sclerosis in patients on peritoneal dialysis. Neth J Med. 2008;66:269-74.
8. Kawanishi H, Moriishi M. Encapsulating Peritoneal Sclerosis: Prevention and treatment. Perit Dial. Int. 2007;27:S289-92.

9. Merkle M, Wornle M. Sclerosis peritonitis: A rare but fatal complication of peritoneal inflammation. Mediators Inflamm. 2012;2012:1-4.

10. Soriano-Cabrera S. Definición y clasificación de los estadios de la enfermedad renal crónica. Prevalencia claves para el diagnóstico precoz. Factores de riesgo de enfermedad renal crónica. Nefrología. 2004;24(S6):27-34.

11. Kawanishi H. The pathogenesis and therapeutic option of encapsulating peritoneal sclerosis. Int J Artif Organs. 2005;28:150-5.

12. Kawanishi H. Encapsulating peritoneal sclerosis. Nephrology 2005;10:249-55.

13. Barrera P, Zambrano P, Contreras A, Dreves P, Salgado I, Vogel A, Encalada R, Cano F. Complicaciones infecciosas en diálisis peritoneal crónica. Rev Chil Pediatr. 2008;79:522-536.

14. Warady B. Consensus guidelines for the treatment of peritonitis in pediatric patients receiving peritoneal dialysis. Perit Dial Int. 2000;20(6):610-24. 